\title{
Hard state neutron star and black hole $X$-ray binaries in the radio:X-ray luminosity plane
}

\author{
Elena Gallo, ${ }^{1 \star}$ Nathalie Degenaar, ${ }^{2}$ and Jakob van den Eijnden ${ }^{2}$ \\ ${ }^{1}$ Department of Astronomy, University of Michigan, 1085 S University, Ann Arbor, MI 48109, USA \\ 2 Anton Pannekoek Institute for Astronomy, University of Amsterdam, Science Park 904, 1098 XH Amsterdam, The Netherlands
}

Accepted XXX. Received YYY; in original form ZZZ

\begin{abstract}
Motivated by the large body of literature around the phenomenological properties of accreting black hole (BH) and neutron star (NS) X-ray binaries in the radio:X-ray luminosity plane, we carry out a comparative regression analysis on $36 \mathrm{BHs}$ and $41 \mathrm{NSs}$ in hard X-ray states, with data over $7 \mathrm{dex}$ in X-ray luminosity for both. The BHs follow a radio to X-ray (logarithmic) luminosity relation with slope $\beta=0.59 \pm 0.02$, consistent with the NSs' slope $\left(\beta=0.44_{-0.04}^{+0.05}\right)$ within $2.5 \sigma$. The best-fitting intercept for the BHs significantly exceeds that for the NSs, cementing BHs as more radio loud, by a factor $\sim 22$. This discrepancy can not be fully accounted for by the mass or bolometric correction gap, nor by the NS boundary layer contribution to the $\mathrm{X}$-rays, and is likely to reflect physical differences in the accretion flow efficiency, or the jet powering mechanism. Once importance sampling is implemented to account for the different luminosity distributions, the slopes of the non-pulsating and pulsating NS subsamples are formally inconsistent $(>3 \sigma)$, unless the transitional millisecond pulsars (whose incoherent radio emission mechanism is not firmly established) are excluded from the analysis. We confirm the lack of a robust partitioning of the BH data set into separate luminosity tracks.
\end{abstract}

Key words: X-rays: binaries - stars: black holes - stars: neutron - methods: statistical

\section{INTRODUCTION}

In spite of recent major advancements in our phenomenological understanding of X-ray binary (XRB) outflows, and their connection with the accretion flow behaviour, the launching mechanism of relativistic jets is still a matter of debate (see, e.g., Fender 2016 for a recent review). While XRBs jets are typically studied in the radio band, where their synchrotron emission dominates over any other mechanism, they are likely launched and accelerated within a few 100s of gravitational radii of the compact object (Gandhi et al. 2017), where the X-ray emission originates. Coordinated radio and X-ray luminosity studies of outbursting XRBs tend to be biased towards the black hole $(\mathrm{BH})$ population. This is likely due to the combination of two main factors. First, the somewhat slower state transition timescales of BH XRBs make it easier to schedule coordinated space and ground observations. Second, and arguably more important, BH XRBs have long been known to be radio brighter than neutron stars (NSs). Seminal work by Fender \& Kuulkers (2001) showed that transient BH XRBs are more radio loud (in terms of their radio to X-ray luminosity ratio, where both luminosities were measured at peak) than transient NSs. At around the same time, Fender \&

* E-mail: egallo@umich.edu
Hendry (2000) noted that the mean radio luminosity of persistent NS Z sources and BHs were broadly consistent with each other, with the persistent Atolls and the transient X-ray pulsars (again at peak) being fainter by a factor of $\gtrsim 5-10$ (the reader is referred to Muñoz-Darias et al. 2014 for recent, detailed reviews on NS X-ray states and nomenclature, and to Belloni \& Motta 2016 for the BHs).

These early studies were concerned with mean and/or peak radio luminosities. Significant advancement came with the onset of multi-wavelength outburst monitoring campaigns (joint with major correlator/receiver upgrades for the Very Large Array and Australia Telescope Compact Array), where a growing number of systems, albeit primarily BHs, have been monitored aggressively in the radio and X-ray band, often over multiple outbursts, and down to very low Eddington ratios. Thanks to these efforts, a coherent phenomenological picture that links distinct X-ray spectral states to different radio properties has emerged for the BHs (Fender 2006; Fender et al. 2009). Broadly speaking, the hard state is associated with (flat/inverted spectrum) radio emission arising from a partially selfabsorbed synchrotron emitting outflow. Compact, hard state radio sources have indeed been resolved into mas-scale collimated jets in two (bright) BHs (GRS1915+205 and Cyg X-1; Dhawan et al. 2000; Stirling et al. 2001), and possibly a NS (Cir X-1; Miller-Jones et al. 2012). As a system enters into outburst, and its X-ray luminosity 


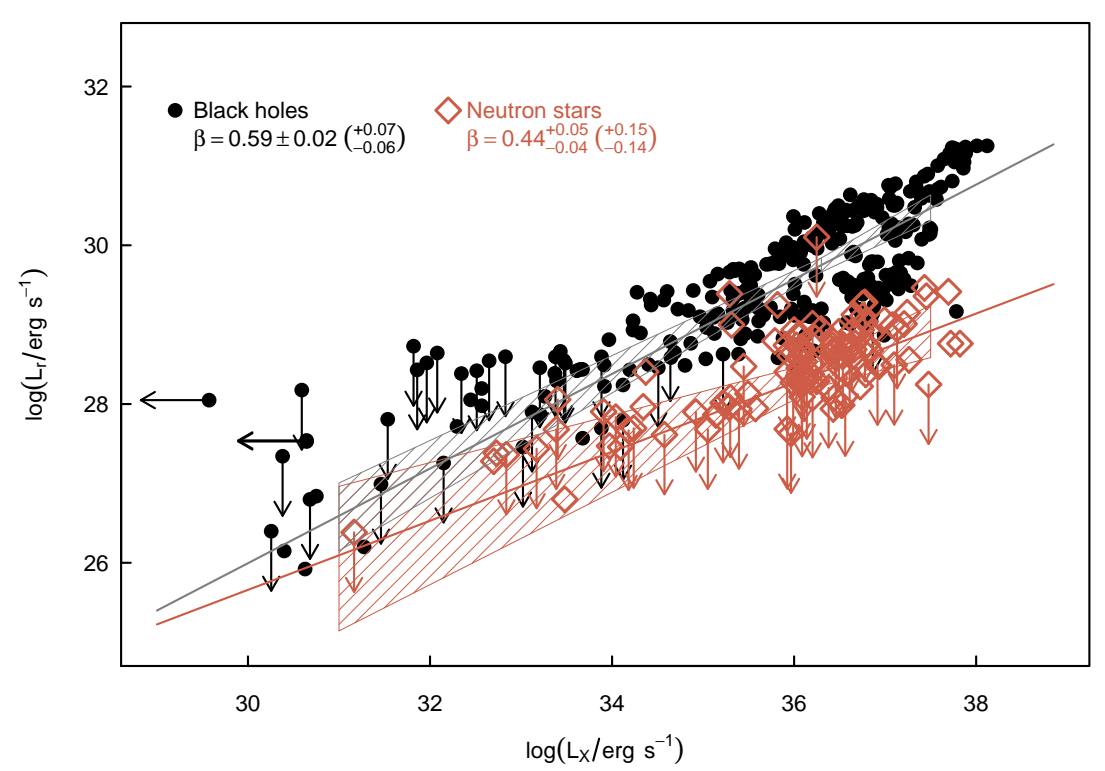

Figure 1. Radio (5 GHz) vs. X-ray (1-10 keV) luminosity for a sample of $36 \mathrm{BHs}$ (dark gray circles) and 41 NSs (orange diamonds) in hard states. Solid lines represent the median of the slope $(\beta)$ posterior distribution, with $3 \sigma$ errors enclosed by the shaded areas. Quoted errors on $\beta$ are at the 1 (3) $\sigma$ confidence level.

starts to increase, so does the radio luminosity of the compact jet. Based on data compiled by Hannikainen et al. (1998) and Corbel et al. (2000), Corbel et al. (2003) first reported on a tight, non linear correlation between the radio and X-ray luminosity of the BH XRB GX339-4 over about eight years covering three major outbursts: $L_{\mathrm{X}} \propto L_{\mathrm{r}}{ }^{0.7}$, over about three orders of magnitude in $L_{\mathrm{X}}$. Shortly thereafter, based on quasi simultaneous radio and X-ray observations of nine more systems, Gallo et al. (2003) argued for a universal radio:X-ray luminosity correlation in the hard state of BH XRBs.

On the NS front, Migliari \& Fender (2006) conducted the first systematic investigation, including data from $\mathrm{Z}$ sources, accreting millisecond X-ray pulsars (AMXPs), and Atoll systems, both in hard and soft states. For the two (Atoll) systems with hard state data (namely, Aql X-1 and 4U 1728-34) they found $L_{\mathrm{X}} \propto L_{\mathrm{r}}{ }^{1.4 \pm 0.2}$, yielding a significantly steeper correlation than found for the BHs, albeit over a significantly narrower dynamic range. Secondly, they confirmed that, whether in absolute or Eddington-scaled units, the "the NSs remain stubbornly less radio loud than the BHs for a given X-ray luminosity", by a factor $\sim 30$. For the reasons discussed above, NS XRB studies have been hampered by the lack of radio detections at X-ray luminosities below $\lesssim 10^{36} \mathrm{erg} \mathrm{s}^{-1}$. With the exception of the so-called transitional millisecond pulsars (tMSPs; Archibald et al. 2009; Papitto et al. 2013), for which a similar correlation to hard state BHs has been claimed over more than three orders of magnitude in $L_{\mathrm{X}}$ (Deller et al. 2015; see, however, Bogdanov et al. 2018), the limited $L_{\mathrm{X}}$ dynamic range makes it challenging to reliably assess the presence of a luminosity correlation for hard state NSs. In an effort to extend previous investigations to lower luminosities/accretion rates, Tetarenko et al. (2016) and Tudor et al. (2017) assembled coordinated radio:X-ray observations of a handful additional hard
Table 1. The dependence of radio luminosity upon X-ray luminosity is parametrized as $\left(\ell_{\mathrm{r}}-28.57\right)=\alpha+\beta\left(\ell_{\mathrm{X}}-36.30\right)$, where $\ell$ denotes logarithmic values in CGS units. The best-fitting intercept $(\alpha)$, slope $(\beta)$, and intrinsic scatter $\left(\sigma_{0}\right)$ are defined as the median of the posterior distribution. Errors are quoted at the $1 \sigma$ c.l.; for $\beta, 3 \sigma$ errors are also given in brackets.

\begin{tabular}{|c|c|c|c|}
\hline Sample & $\alpha$ & $\beta$ & $\sigma_{0}$ \\
\hline $\mathrm{BH}$ & $+1.18_{-0.03}^{+0.03}$ & $0.59_{-0.02}^{+0.02}\left(\begin{array}{l}+0.07 \\
-0.06\end{array}\right)$ & $0.46_{-0.02}^{+0.02}$ \\
\hline NS & $-0.17_{-0.05}^{+0.05}$ & $0.44_{-0.04}^{+0.05}\left({ }_{-0.13}^{+0.15}\right)$ & $0.43_{-0.04}^{+0.05}$ \\
\hline NS-tMSP & $-0.18_{-0.05}^{+0.05}$ & $0.49_{-0.05}^{+0.06}\left({ }_{-0.15}^{+0.22}\right)$ & $0.41_{-0.04}^{+0.05}$ \\
\hline Atoll & $-0.25_{-0.06}^{+0.06}$ & $0.71_{-0.09}^{+0.11}\left({ }_{-0.24}^{+0.36}\right)$ & $0.30_{-0.04}^{+0.05}$ \\
\hline AMXP & $-0.28_{-0.15}^{+0.14}$ & $0.27_{-0.10}^{+0.09}\left({ }_{-0.33}^{+0.29}\right)$ & $0.59_{-0.09}^{+0.12}$ \\
\hline AMXP-tMSP & $-0.33_{-0.16}^{+0.14}$ & $0.19_{-0.14}^{+0.13}\left({ }_{-0.50}^{+0.45}\right)$ & $0.56_{-0.10}^{+0.14}$ \\
\hline w1 Atoll & $-0.23_{-0.11}^{+0.09}$ & $1.16_{-0.24}^{+0.28}\left({ }_{-0.59}^{+0.91}\right)$ & $0.29_{-0.10}^{+0.12}$ \\
\hline w2 Atoll & $-0.18_{-0.08}^{+0.07}$ & $1.39_{-0.30}^{+0.35}\left(\begin{array}{l}+1.36 \\
-1.02\end{array}\right)$ & $0.21_{-0.09}^{+0.11}$ \\
\hline
\end{tabular}

state systems (including new data for the globular cluster Atoll EXO 1745-248, three AMXPs, and the non-pulsating system Cen $\mathrm{X}-4$, both in quiescence as well as in outburst, plus a re-analysis of $4 \mathrm{U}$ 1728-34 and Aql X-1 data). Their results point toward a complex pattern of behaviour, with different systems exhibiting different degrees of disc-jet coupling.

In this Letter, we present the largest yet collection of quasisimultaneous radio:X-ray observations of hard state $\mathrm{BH}$ and NS XRBs. A broader study will be presented in a companion work (Van den Eijnden et al., in prep., including thermal and very high state BHs, Z sources, soft-state Atolls, and slowly pulsating NSs). We start by collating data points from Gallo et al. (2014), for BHs, and Migliari \& Fender (2006) plus Migliari et al. (2011), for the 


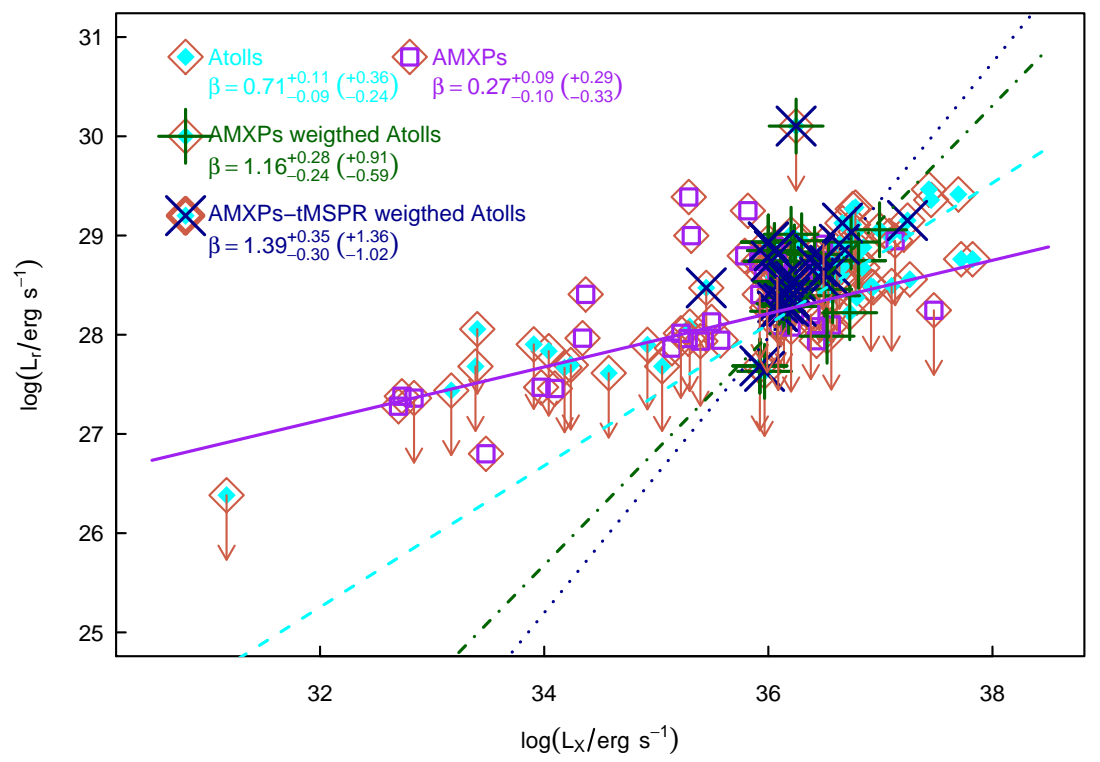

Figure 2. $L_{\mathrm{X}}$ distribution-weighted NS regression analysis, with weighting functions defined in Fig. 3. Quoted errors on $\beta$ are at the 1 (3) $\sigma$ confidence level.

NSs. Additional data points come from further literature searches and or new data collected by our group. For the NSs, we select data for hard state Atolls and AMXPs, including the 3 known tMSPs. In the case of reported radio fluxes with no accompanying, dedicated X-ray coverage, X-ray fluxes around the time of the radio observation(s) are obtained from the 1-day averaged all-sky monitors on board the Rossi X-ray Timing Explorer (RXTE) or The Monitor of All-sky X-ray Image (MAXI). X-ray and radio fluxes are converted to unabsorbed 1-10 keV X-ray luminosities and $5 \mathrm{GHz}$ luminosities, respectively. A flat radio spectrum is assumed for the purpose of converting radio flux densities to $5 \mathrm{GHz}$ radio luminosity. Overall, our sample is composed of $36 \mathrm{BH}$ and $41 \mathrm{NS}$ systems fainter than $L_{\mathrm{X}} \lesssim 10^{38} \mathrm{erg} \mathrm{s}^{-1}$; data points, along with a list of adopted distances and references, can be downloaded from: https : // jakobvdeijnden. wordpress.com/radioxray/.

\section{REGRESSION ANALYSIS}

We start by assessing whether the distribution of $\ell_{\mathrm{X}}$ for the NSs (109 data points) is consistent with being drawn from that of the BHs (306 data points, inclusive of $3 \mathrm{X}$-ray upper limits), where $\ell$ denotes logarithmic luminosities in units of $\mathrm{erg} \mathrm{s}^{-1}$. A two-sample Kolmogorov-Smirnov (KS) test probability $p=0.04$ implies that the two distributions are marginally consistent. For each sample, we parameterize the dependence of radio upon X-ray luminosity as $\left(\ell_{\mathrm{r}}-28.57\right)=\alpha+\beta\left(\ell_{\mathrm{X}}-36.30\right)$, with intrinsic random scatter included in the fitting. Centering is based on the median luminosities for the combined sample. We run the Bayesian modeling routine described by Kelly (2007) and implemented in IDL as
LINMIX_ERR.PRO, which enables us to include censored data in the analysis. In line with previous work (Gallo et al. 2012, 2014), we assume 0.15 dex uncertainties in both $\ell_{\mathrm{X}}$ and $\ell_{\mathrm{r}}$, with Gaussian likelihood functions on $\ell_{\mathrm{r}}$ and $\ell_{\mathrm{X}}$, and uniform probability below upper limits. For the purpose of estimating the most likely values of the correlation intercept $(\alpha)$, slope $(\beta)$, and intrinsic scatter $\left(\sigma_{0}\right)$, we calculate the median of 10,000 draws from the posterior distributions; $1 \sigma$ confidence errors are calculated as the 16-84th percentiles of the posterior distributions (Table 1). Fig. 1 illustrates the results from the $\mathrm{BH}$ vs. NS regression analysis: the $\mathrm{BH}$ data points follow a relation with slope $\beta=0.59 \pm 0.02$, intercept $\alpha=+1.18 \pm 0.03$, and large intrinsic scatter, $\sigma_{0}=0.46 \pm 0.02$. For the NSs, $\beta=0.44_{-0.04}^{+0.05}$, $\alpha=-0.17 \pm 0.05$, and $\sigma_{0}=0.43_{-0.04}^{+0.05}$. Whereas the two correlation slopes are consistent with each other within $2.5 \sigma$, the best-fitting $\mathrm{BH}$ intercept exceeds that for the NS by 1.35 dex, confirming the BHs as substantially more radio-loud, by a factor $\sim 22$.

A separate question we wish to address is whether there is any statistically significant difference among different classes of NSs. We start by comparing the sub-sample of X-ray pulsating systems (i.e. the AMXPs, including the 3 tMSPs, for a total of 38 data points) to the (non-pulsating) Atolls (71 data points). Taken at face value, AMXPs are best described by a shallow relation, with slope $\beta=$ $0.27_{-0.10}^{+0.09}$, whereas $\beta=0.71_{-0.09}^{+0.11}$ for the Atolls (solid purple and dashed cyan lines, respectively, in Fig 2). Although the slopes are consistent within $3 \sigma$ (see Table 1), this comparison is intrinsically flawed. A two-sample KS test rules out with high confidence that the two $\ell_{\mathrm{X}}$ distributions are consistent with each other. To control for this, we conduct a weighted comparison by drawing random subsamples from the Atoll population, weighted to match the AMXP probability density distributions (PDFs) as weighting functions (Fig. 

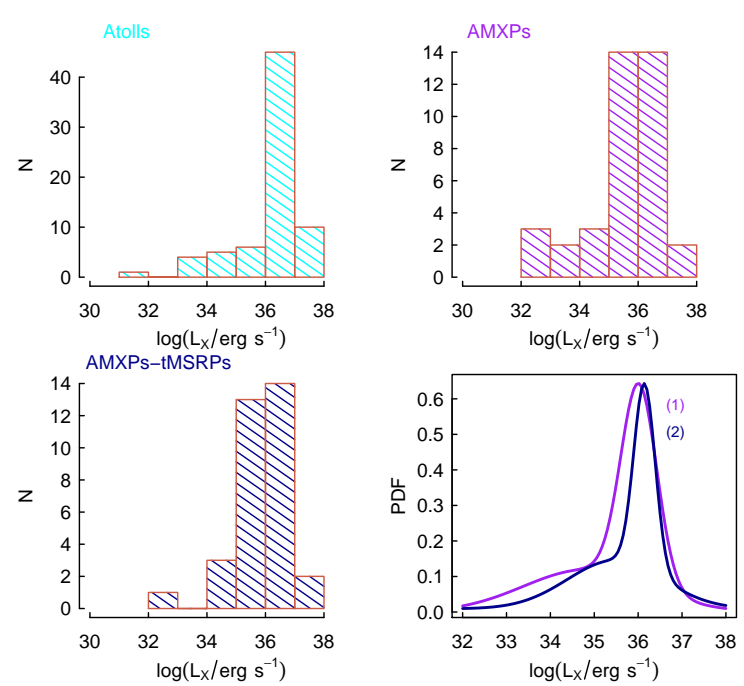

Figure 3. X-ray luminosity distributions for the different NS sub-populations: Atolls, AMXPs and tMSPs. The comparative regression analysis is carried out through importance sampling, i.e. using the AMXP (1) and AMXP-tMSP (2) probability density distributions (PDF) as weighing functions.

3 ), including and excluding the tMSPs. The AMXP-weighted Atoll sub-sample (denoted as w1-Atolls in Table 1) yields $\beta=1.16_{-0.24}^{+0.28}$ (dashed-dotted green line in Fig. 2), which is formally inconsistent $(>3 \sigma)$ with the AMXP's slope (the significantly steeper slope of the AMXP-weighted Atolls with respect to the full Atoll sample can be understood noting that the majority of the data points at $\ell_{\mathrm{X}} \gtrsim 36$ are excluded by the importance sampling). The same exercise is carried out excluding the 3 tMSPs ( 5 data points) from the AMXPs population. Controlling for the different $\ell_{\mathrm{X}}$ distribution as above (i.e. using PDF 2 in Fig. 3 as a weighing function), we find that the (AMXP-tMSP)-weighted Atoll distribution (w2 Atolls in Table 1) is best described by $\beta=1.39_{-0.30}^{+0.35}$ (dotted blue line in Fig. 2), which is consistent with the (AMXP-tMSP)'s slope $\left(0.19_{-0.14}^{+0.13}\right)$ within $2.5 \sigma$.

This begs the question whether the NS vs. BH comparison, too, is affected by the inclusion of the tMSPs. A two-sample KS test shows that, after the exclusion of tMSPs from the NS sample, the NS and $\mathrm{BH} \ell_{\mathrm{X}}$ distributions are still consistent with each other $(p=0.05$ ), so no importance weighting is required. Re-fitting (NStMSP) sample yields a best-fitting slope $\beta=0.49_{-0.05}^{+0.06}$, which is still consistent with the BHs' within $2 \sigma$.

Last, we apply the same formalism described in Gallo et al. $(2012,2014)$ to assess whether the BH sample is best described by a single vs. two or more clusters of data points (this approach is intrinsically limited to radio and X-ray detections only). After normalizing the axes to standardized coordinates and rotating them to unit variance, we run the clustering algorithms "affinity propagation" (APCLUST; Frey \& Dueck 2007), and HYBRIDHCLUST (Chipman \& Tibshirani 2006). At face value, both prefer a two-cluster description of the data (left and middle panel in Fig. 4). Interestingly, both algorithms identify nearly parallel tracks, rather than a main track across the entire $\ell_{\mathrm{X}}$ dynamic range, plus a steeper track at $\ell_{\mathrm{X}} \gtrsim 35$ (see, e.g. Coriat et al. 2011). The actual cluster membership, however, is not robust. Additionally, after scrambling the data points with a Gaussian distribution of width $\sigma \gtrsim 0.15$, different cluster centres and group memberships are identified. The lack of a reliable partitioning is confirmed by the fact that the median radio loudness (here defined as $\ell_{\mathrm{r}}-\ell_{\mathrm{X}}$ ) for individual systems (calculated above $\ell_{\mathrm{X}}=36.3$, i.e. in the range where two diverging tracks are often claimed), while consistent with a multi-modal distribution (Fig. 4, right panel), does not reflect any of the above clustering analysis results. To summarize, our analysis confirms the lack of a robust partitioning in the BH sample, defined as the ability to reliably and independently define two distinct tracks (this does not, however, constitute proof that the $\mathrm{BH}$ data are best described by a single distribution). Owing to this uncertainty, no multi-track regression analysis is warranted for the $\mathrm{BH}$ sample.

\section{DISCUSSION}

As discussed by Tudor et al. (2017), individual NS systems exhibit a broad and complex range of behaviour in the radio:X-ray luminosity plane, including anti-correlations (albeit over a limited dynamic range). Additionally, the nature of the incoherent radio emission from tMSPs, i.e. the only NSs with radio detections below $\ell_{\mathrm{X}} \lesssim 35$, is still a matter of debate (Bogdanov et al. 2018). Our result that the Atoll and AMXP populations are described by formally consistent scaling relations only if the tMSPs are excluded from the weighted regression analysis goes to show how these kinds of investigations hinge on properly accounting for the different luminosity distributions, and further stresses the importance of unraveling the tMSP radio emission mechanism during the $\mathrm{X}$-ray pulsating mode.

Regarding the $\mathrm{BH}$ sample, we confirm the conclusions by Gallo et al. (2014) that no robust partitioning into two or more clusters (or tracks) can be identified. Several authors have investigated the possible origin of the so-called radio-quiet BH track (Soleri \& Fender 2011; Dinçer et al. 2014; Meyer-Hofmeister \& Meyer 2014; Drappeau et al. 2015; Espinasse \& Fender 2018). While it is entirely possible that the large scatter about the correlation, particularly above $10^{35} \mathrm{erg} \mathrm{s}^{-1}$, can indeed arise from an enhanced disc contribution to the X-ray signal, or somewhat steeper radio spectral indices, the formal divide between the so-called standard and radio quiet track is rather blurred, with our latest clustering analysis indicating two nearly parallel tracks. Additionally, in spite of numerous claims to the contrary, the scatter about the NS relation is as large as that of the BHs.

This study confirms the enhanced radio-loudness of the $\mathrm{BH}$ population across a broad dynamic range in X-ray luminosity. The factor of $\gtrsim 5$ difference in the accretor mass can not possibly entirely account for the discrepancy: (naively) correcting for the mass term using the Fundamental Plane of Black Hole Activity relation ${ }^{1}$ yields a difference of 0.81 dex in the best-fitting intercept, implying that the BHs remain more radio loud, by a factor $\sim 6.5$, after accounting for the mass term. The gap could be further reduced if the total (as opposed to 1-10 keV) accretion luminosity were considered: such bolometric correction can be as high as 5-8 for the BHs (Zdziarski et al. 2004), vs. a factor 2-3 for the NSs (Galloway et al. 2008). Adding this to the mass term correction would reduce

1 Where we adopt the best-fitting parameters of Merloni et al. (2003) and use compact object masses from Casares et al. (2017), when available, or 2 vs. $10 M_{\odot}$ for the NSs and BHs, respectively, otherwise. 


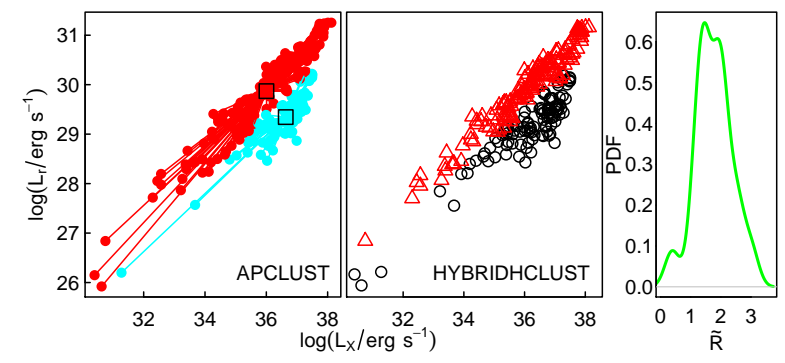

Figure 4. Clustering analysis on the $\mathrm{BH}$ data points (detections only): both APCLUST (left) and HYBRIDHCLUST (centre) return a two cluster partitioning, albeit with slightly different membership. Right: PDF of the median radio loudness for individual systems, calculated above $\ell_{\mathrm{X}}=36.2$.

the radio loudness difference to a factor of 3. Finally, contribution to the quoted 1-10 keV luminosities from the NS boundary layer up to between 30 to 50 per cent of the total X-ray luminosity (Burke et al. 2017) could further reduce the BH-to-NS radio loudness ratio to a minimum of 2.5 (notice that this is obtained by conservatively adopting the largest possible "colour" corrections in all of the above cases). This can be equally interpreted as the NSs being significantly brighter in $\mathrm{X}$-rays than the $\mathrm{BHs}$ at a given radio luminosity. Advection of energy across the $\mathrm{BH}$ event horizon (Narayan \& Yi 1995 ) is unlikely to be responsible for this difference, as one would expect the BHs to become progressively X-ray dimmer with respect to the NSs towards lower X-ray luminosities, which, in turn, would yield inconsistent (at the $>3 \sigma$ level) slopes. Whereas increasing evidence has solidified the discrepancy in radio loudness between $\mathrm{BH}$ and NS XRBs, its origin remains unclear. Spin-powering of BH jets remains an appealing explanation, albeit several studies on the matter have failed to reach a compelling conclusion (Fender et al. 2010; Narayan \& McClintock 2012; Russell et al. 2013; Steiner et al. 2013). Additionally, it has to be noted that the radio luminosity (in and of itself a poor indicator of total jet power) of NSs in soft X-ray states can approach that of the BHs. This will be further investigated in a companion paper (Van den Eijnden et al., in prep.).

\section{ACKNOWLEDGEMENTS}

ND and JvdE are supported by a Vidi grant from NWO awarded to ND. We are grateful to James Miller-Jones, Rich Plotkin and Tom Russell for sharing their radio data. We thank the referee for a prompt and insightful report.

\section{REFERENCES}

Archibald A., et al., 2009, Science, 324, 1411

Belloni T. M., Motta S. E., 2016, in Bambi C., ed., Astrophysics and Space Science Library Vol. 440, Astrophysics of Black Holes: From Fundamental Aspects to Latest Developments. p. 61 (arXiv:1603.07872)

Bogdanov S., et al., 2018, ApJ, 856, 54

Burke M. J., Gilfanov M., Sunyaev R., 2017, MNRAS, 466, 194
Casares J., Jonker P. G., Israelian G., 2017, preprint, (arXiv:1701.07450)

Chipman H., Tibshirani R., 2006, Biostatistics, 7, 286

Corbel S., Fender R. P., Tzioumis A. K., Nowak M., McIntyre V., Durouchoux P., Sood R., 2000, A\&A, 359, 251

Corbel S., Nowak M. A., Fender R. P., Tzioumis A. K., Markoff S., 2003, A\&A, 400, 1007

Coriat M., et al., 2011, MNRAS, 414, 677

Deller A. T., et al., 2015, ApJ, 809, 13

Dhawan V., Mirabel I. F., Rodríguez L. F., 2000, ApJ, 543, 373

Dinçer T., Kalemci E., Tomsick J. A., Buxton M. M., Bailyn C. D., 2014, ApJ, 795, 74

Drappeau S., Malzac J., Belmont R., Gandhi P., Corbel S., 2015, MNRAS, 447, 3832

Espinasse M., Fender R., 2018, MNRAS, 473, 4122

Fender R., 2006, Jets from X-ray binaries, 1 edn. Cambridge University Press, pp 381-419

Fender R., 2016, Astronomische Nachrichten, 337, 381

Fender R. P., Hendry M. A., 2000, MNRAS, 317, 1

Fender R. P., Kuulkers E., 2001, MNRAS, 324, 923

Fender R. P., Homan J., Belloni T. M., 2009, MNRAS, 396, 1370

Fender R. P., Gallo E., Russell D., 2010, MNRAS, 406, 1425

Frey B. J., Dueck D., 2007, Science, 315, 972

Gallo E., Fender R. P., Pooley G. G., 2003, MNRAS, 344, 60

Gallo E., Miller B. P., Fender R., 2012, MNRAS, 423, 590

Gallo E., et al., 2014, MNRAS, 445, 290

Galloway D. K., Muno M. P., Hartman J. M., Psaltis D., Chakrabarty D., 2008, ApJS, 179, 360

Gandhi P., et al., 2017, Nature Astronomy, 1, 859

Hannikainen D. C., Hunstead R. W., Campbell-Wilson D., Sood

R. K., 1998, A\&A, 337, 460

Kelly B. C., 2007, ApJ, 665, 1489

Merloni A., Heinz S., di Matteo T., 2003, MNRAS, 345, 1057

Meyer-Hofmeister E., Meyer F., 2014, A\&A, 562, A142

Migliari S., Fender R. P., 2006, MNRAS, 366, 79

Migliari S., Miller-Jones J. C. A., Russell D. M., 2011, MNRAS, 415, 2407

Miller-Jones J. C. A., et al., 2012, MNRAS, 419, L49

Muñoz-Darias T., Fender R. P., Motta S. E., Belloni T. M., 2014, MNRAS, 443, 3270

Narayan R., McClintock J. E., 2012, MNRAS, 419, L69

Narayan R., Yi I., 1995, ApJ, 452, 710

Papitto A., et al., 2013, Nature, 501, 517

Russell D. M., Gallo E., Fender R. P., 2013, MNRAS, 431, 405

Soleri P., Fender R., 2011, MNRAS, 413, 2269

Steiner J. F., McClintock J. E., Narayan R., 2013, ApJ, 762, 104

Stirling A. M., Spencer R. E., de la Force C. J., Garrett M. A., Fender R. P., Ogley R. N., 2001, MNRAS, 327, 1273

Tetarenko B. E., et al., 2016, ApJ, 825, 10

Tudor V., et al., 2017, MNRAS, 470, 324

Zdziarski A. A., Gierliński M., Mikołajewska J., Wardziński G., Smith D. M., Harmon B. A., Kitamoto S., 2004, MNRAS, 351, 791

MNRAS 000, 1-?? (2018) 